\title{
Text Line Segmentation for Unconstrained Handwritten Document Images Using Neighborhood Connected Component Analysis
}

\author{
Abhishek Khandelwal ${ }^{1}$, Pritha Choudhury ${ }^{1}$, Ram Sarkar $^{2}$, Subhadip Basu ${ }^{2}$, \\ Mita Nasipuri ${ }^{2}$, and Nibaran Das ${ }^{2}$ \\ ${ }^{1}$ CSE Department, Sikkim Manipal Institute of Technology, Sikkim, India \\ ${ }^{2}$ CSE Department, Jadavpur University, Kolkata, India
}

\begin{abstract}
Text line extraction is the first and one of the most critical steps in optical character recognition (OCR) of unconstrained handwritten documents. The present work reports a new methodology based on comparison of neighborhood connected components to determine whether they belong to the same text line. Components which are very small or very large compared to the average component height are ignored in the preprocessing step. During postprocessing, such components are reconsidered and allocated to the lines to which they most suitably belong. The performance of the developed technique is evaluated on the benchmark training dataset for the ICDAR 2009 handwriting segmentation contest. The dataset consists of English, French, German and Greek handwritten texts. The overall text line identification accuracy on the mentioned dataset is observed to be around $93.35 \%$.
\end{abstract}

Keywords: Text line identification, handwritten script, neighborhood connected component analysis.

\section{Introduction}

Identification of text lines is the first and most important step in the process of optical character recognition (OCR) of handwritten document images. If line identification is not accurate, (for example, if two or more lines are merged) then none of the words and consequently none of the characters in the constituent lines can be identified correctly. The same problem occurs if a line is erroneously split into two or more parts. Such scenarios are unacceptable for large-scale recognition of handwritten documents.

Previous techniques using Hough transform consider a set of points of initial image as input while the lines that fit best to these points are calculated. These methods vary in the set of points considered for voting procedure, viz. gravity centers [1] or minima points [2] of the connected components. A recent block based Hough Transform method [3] takes into account gravity centers of parts of CCs.

Smearing methods include the Fuzzy RLSA [4], in which the value of each pixel is the sum of all pixels in the original image within a specified horizontal distance. Adaptive RLSA [5] evolves from Classical RLSA [6] and uses additional smoothing 
constraints in regard to the geometrical properties of neighboring CCs. Another technique that uses morphological operations and RLSA to segment text lines from unconstrained handwritten document images is described in [7].

An MST based clustering technique [8] with distance metric learning has been suggested for text line identification in Chinese handwritten documents. A technique described in [9] makes use of Mumford-Shah (MS) model; line segmentation in this technique is achieved by minimization of MS energy. A technique described in [10] uses density estimation and level set methods for text line extraction. A novel technique using hypothetical water flows at specific angles from both sides of the document image for text line extraction is described in [11].

In this paper, we present an effective alternative technique for identifying text lines in handwritten documents. As a first step, we have implemented an eight-way connected-component-labeling (CCL) algorithm to identify the most basic elements in the text document as unique objects. During preprocessing, we ignore components identified as noise. Some very large components might be formed as a result of overlap of two or more elements belonging to adjacent lines. Such components are split. Thus, a final set of components is derived for text line identification. Post-processing steps include reconsidering the components previously ignored as noise. Some of these might have been actually been small handwritten parts of text and such components are allocated to suitable lines.

\section{The Present Work}

The scanned handwritten document image is first binarized, with each foreground data pixel is represented by label ' 1 ' and each background pixel is represented by label ' 0 '. All the following steps are then implemented on these binarized data file.

\subsection{Preprocessing of Scanned Document Images}

Preprocessing of the text document in order to phase out noise and outlier data is very important for identification of text lines correctly. During scanning of handwritten documents, many small dot-like noisy elements may appear on the scanned image. It has been observed that these elements are usually of a size much smaller than the smallest components in the handwritten documents. So, two threshold values, $\mathrm{T}_{1}$ subjective to the height of the document image, and $T_{2}$ subjective to the width of the document image have been set. A component with a height less than $T_{1}$ and width less than $T_{2}$ is ignored, while creating the set of uniquely labeled components to be considered for text line identification. In this process, some very small components belonging to the actual handwritten text might also get ignored erroneously, but they shall be reconsidered and merged into suitable text lines during refinement of the identified text lines at a later stage of the algorithm.

Another type of noise that may appear on the document image while scanning, is long lines at the edges of the documents. These lines shall interfere in the implementation of the current work severely, and hence must be removed while preprocessing 
the document image. Components having a height greater than $1 / 4^{\text {th }}$ the page height or width greater than $1 / 3^{\text {rd }}$ the page width are identified as such noise elements and are ignored for subsequent processing.

\subsection{Analysis of Identified Components}

An eight-way Connected Component Labeling (CCL) algorithm is implemented to uniquely label and build the set of components to be considered for text line identification. For analysis of identified components, first the average component height $H_{\text {Cavg }}$ and average component width $W_{\text {Cavg }}$ is computed as follows:

Let $\mathrm{N}$ be the total number of components, then the component height takes values from the finite data set $x_{1}, x_{2}, \ldots, x_{N}$. Let the arithmetic mean of the heights of the components be $\mu$. Then,

$$
\sigma=\sqrt{\frac{1}{N} \sum_{i=1}^{N}\left(x_{i}-\mu\right)^{2}},
$$

Only components with height ranging from $\mu-\sigma$ to $\mu+\sigma$ are considered for calculating the final average component height $H_{\text {Cavg }}$, which is the arithmetic mean of all such values. The average component width $W_{\text {Cavg }}$ is computed in a similar fashion.

It has been observed in some cases of handwritten text that a character belonging to a particular line may touch another character belonging to an upper or lower line. These two characters are identified as a single component by CCL algorithm, and have a height much larger than the average height $H_{\text {Cavg }}$ which is computed using the above mentioned method. Hence it becomes necessary that this component be split into its constituent characters so that two or more lines are not merged in the step of line identification. In the present work, components having a height greater than $\delta * H_{\text {Cavg }}$ have been split horizontally into two equal halves; where $\delta$ is a heuristically chosen tuning parameter. Here it has been assumed that not more than two consecutive text lines touch each other. Thus a final set $\{S\}$ of components is constructed for formation of lines from the scanned document image.

\subsection{Neighborhood Formation and Text Line Identification}

For each component $C_{i}$, a general neighborhood $N_{C i}$ is defined, and a component under consideration looks for candidate components in its neighborhood to determine whether any such component belongs to the same line as the current component. The neighborhood $N_{C i}$ is defined with respect to the average component width $W_{\text {Cavg }}$ and average component height $H_{\text {Cavg }}$ computed previously. It is a rectangular area of width $\alpha^{*} W_{\text {Cavg }}$ and height $\beta^{*} H_{\text {Cavg }}$ with the dimensional center of the component $C_{i}$ as the centre of the rectangle; where $\alpha$ and $\beta$ are experimentally chosen tuning parameters.

Each component $C_{i}$ within the set $\{S\}$ is considered in sequence. Let $\left\{C_{i N}\right\}$ be the set of components in the neighborhood $N_{C i}$ of $C_{i}$. The component $C_{i}$ looks for neighborhood components which satisfy at least one of the following conditions: 
i) $C_{i}$ spans at least a fraction $\eta_{1}$ of the height of $C_{i j} ; \mathrm{j} \in \mathrm{N}$, height wise or vice versa.

ii) The height-wise midpoints of $C_{i}$ and $C_{i j} ; \mathrm{j} \in \mathrm{N}$, have a vertical distance less than a threshold $\eta_{2}$ of the height of $C_{i}$ or $C_{i j}$.

Here $\eta_{1}$ and $\eta_{2}$ are experimentally chosen tuning parameters. A component $C_{i j}$, found to satisfy either or both of these conditions, is allocated to the line to which $C_{i}$ belongs. The bounds of the line to which $C_{i}$ belongs, are initialized with the corresponding bounds of $C_{i}$ and the area of the line keeps increasing as more components are allocated to the same line. A component $C i j$, which has already been allocated to a line, shall not be considered for future comparisons with any other component for identification of text lines.

While forming the set $\{S\}$ of components for text line identification we have ignored some small components which were considered to be noisy data. Such components may be ascendants/descendants or very small characters belonging to text lines thus identified. We thus consider all such components one by one and try to allocate them to the lines to which they most suitably belong.

For a particular text line, we consider all such components which lie partially or completely within its bounding box. We then label these components with the same label as that of the text line. This step fairly allocates small components into the text lines of which they should be a part.

\section{Experimental Results}

We have adopted a means of performance evaluation which has already been used in ICDAR 2003, 2005 and 2007 page segmentation contests, and ICDAR 2007 and 2009 handwriting segmentation contests $[3,5]$.

In this method, the segmentation result is compared to an already annotated ground truth. It is based on counting the number of matches between the generated segmentation results and the existing ground truth. The performance of our present work was evaluated on the training set of handwritten documents used in ICDAR handwriting segmentation contest 2009. The test set consisted of 100 pages of handwritten texts in English, French, German, and Greek. These documents were either historical documents or modern texts written by different individuals, not containing any images, logos, etc. The text in most historical documents was highly fragmented. Most of these handwritten documents had skewed/curvy text lines. For all these document images, we had the ground truth data files for comparison with our segmentation results. The documents were scanned at 300dpi and had a typical resolution of 2500x2000 pixels.

The experimental values for the tuning parameters, mentioned in our technique, were set to: $\delta=2.7, \alpha=7, \beta=5, \eta_{1}=0.6, \eta_{2}=0.6$. It is worth noting that our proposed method gives a Detection Rate (DR) of $94.0 \%$, Recognition Accuracy (RA) of 92.7\%, and hence F-Measure (FM) of $93.35 \%$ which outperforms most of the orthodox approaches for text line identification in handwritten documents.

Figs. 1-2 show results of the developed technique on sample test pages, along with the ground truth data. The bounding boxes of the text lines have been highlighted for ease of identification of the same. 


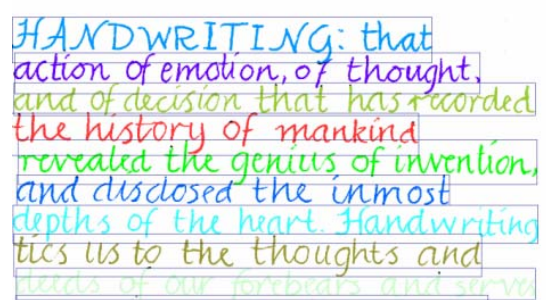

humanity. No machine or technology can replace the contribution or continuing importance of this skill. It has been neecessary in to civilization as our next breath.

$$
\text { - Michael R. Sull- }
$$

(a) Ground truth data

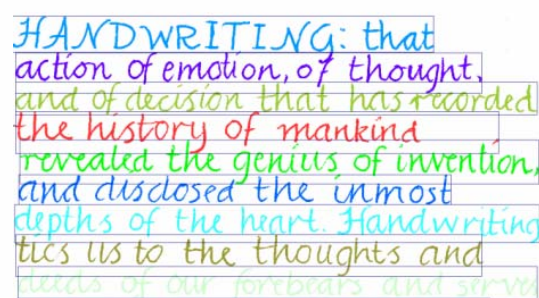

humanity. No machine or technology can replace the contribution or continuing importance of this skill. It has been neccssary in every age and is jus as vital breath.

\section{- Michael R. Sull-}

Fig. 1. Identification results of the developed technique on a page with very close text lines

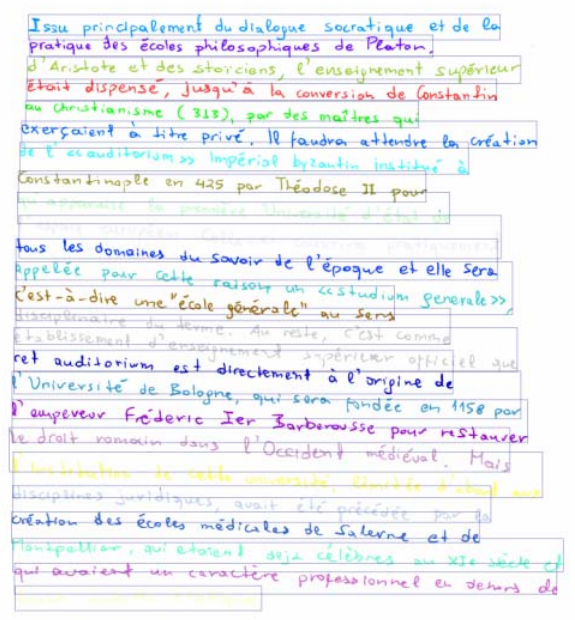

(a) Ground truth data

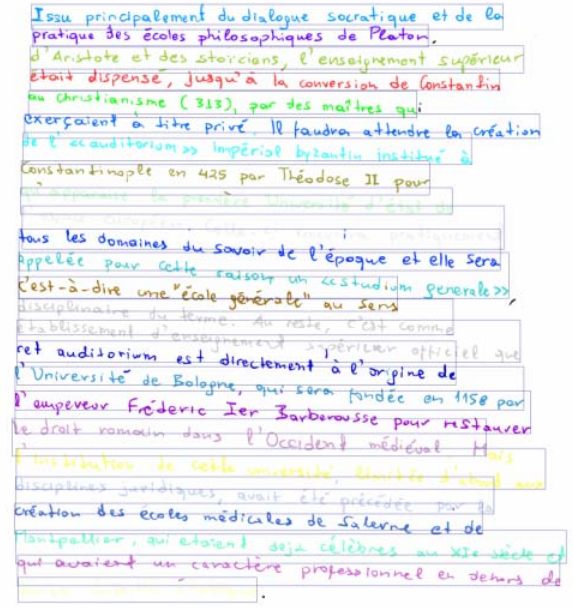

(b) Generated output

Fig. 2. Identification results of the developed technique on a page with skew and extraneous ascendants/descendants

\section{Conclusion}

In this paper, we have developed an effective technique for identification of text lines in handwritten documents. The method uses a novel approach of height-specific dimensional comparison of neighborhood components to classify them into text lines. A post-processing step based on a Euclidean distance metric efficiently classifies small 
components into text lines to which they most suitably belong. Although the performance of the developed methodology was evaluated on English, French, Greek and German, it can also be applied to various other scripts.

Future work may involve segmentation of text lines into constituent words. It also concerns improvement of the technique used for splitting components into constituent words/characters belonging to different lines. Inclusion of a skew-correction step just before the actual identification of text lines would improve the performance of the key modules by a significant amount. Small components ignored during preprocessing can be allocated to suitable lines using an improved technique. In a nutshell, the work presented here outlines the technicalities of an effective method for identification of text lines from unconstrained handwritten document pages.

\section{Acknowledgement}

Authors are thankful to the CMATER, SRUVM, CSE Department, Jadavpur University, for providing infrastructural facilities during progress of the work.

\section{References}

1. Likforman, L., et al.: A Hough based algorithm for extracting text lines in handwritten documents. In: Proc. of the Third ICDAR, Montreal, Canada, pp. 774-777 (1995)

2. $\mathrm{Pu}, \mathrm{Y}$., et al.: A natural learning algorithm based on Hough transform for text lines extraction in handwritten documents. In: Proc. of the 6th IWFHR, pp. 637-646 (1998)

3. Louloudis, G., et al.: A block-based Hough transform mapping for text line detection in handwritten documents. In: The 10th IWFHR, France, October 2006, pp. 515-520 (2006)

4. Shi, Z., et al.: Line separation for complex document images using fuzzy run-length. In: First International Workshop on Document Image Analysis for Libraries, p. 306 (2004)

5. Gatos, B., et al.: ICDAR2007 Handwriting Segmentation Contest. In: the Ninth ICDAR, Curitiba, Brazil, September 2007, pp. 1284-1288 (2007)

6. Wahl, F.M., et al.: Block segmentation and text extraction in mixed text/image documents. Computer Graphics and Image Processing 20, 375-390 (1982)

7. Roy, P.P., et al.: Morphology Based Handwritten Line Segmentation Using Foreground and Background Information. In: Proc. of ICFHR, Canada, pp. 241-246 (2008)

8. Yin, F., et al.: Handwritten Text Line Segmentation by Clustering with Distance Metric Learning. In: Proc. of ICFHR, Canada, August 91-21, pp. 229-234 (2008)

9. Du, X., et al.: Text Line Segmentation in Handwritten Documents Using Mumford-Shah Model. In: Proc. of ICFHR, Canada, August 91-21, pp. 253-258 (2008)

10. Li, Y., et al.: Script-Independent Text Line Segmentation in Freestyle Handwritten Documents. IEEE Transactions on PAMI 30(8), 1313-1329 (2008)

11. Basu, S., et al.: Text line extraction from multi-skewed handwritten documents. Pattern Recognition 40(6), 1825-1839 (2007) 